\title{
Positional Regulation of Electrostatic Micro-electromechanical Actuator via Adaptive Two-stage Sliding Mode Control
}

\author{
Chi-Hsin Yang, ${ }^{1}$ Kun-Chieh Wang, ${ }^{1 *}$ and Long $\mathrm{Wu}^{1,2}$ \\ ${ }^{1}$ School of Mechanical and Electric Engineering, Sanming University, Sanming 365004, Fujian Province, China \\ ${ }^{2}$ Fujian Public Service Platform of Mini-EREV Power System, Sanming University, \\ Sanming 365004, Fujian Province, China
}

(Received March 31, 2020; accepted August 7, 2020)

Keywords: micro-electromechanical (MEM) actuator, positional regulation, adaptive, sliding mode control

In this study, we examine an adaptive two-stage sliding mode control scheme for an electrostatically actuated micro-electromechanical (MEM) actuator. The objective of the control scheme is to regulate the position of the movable plate in the MEM actuator by voltage control. For accurate measurements of system states, direct detection is possible by means of an existing sensing technique. Two main steps are applied to design the closed-loop control system. Firstly, for the dynamical model of the MEM actuator, a normalized time-scale approach and a nonlinear state transformation are introduced. Then, the adaptive two-stage sliding mode control is designed to achieve the control goal. The stability of the closed-loop control is proven and numerical studies to verify the control scheme are also performed.

\section{Introduction}

Nowadays, the electrostatic micro-electromechanical (MEM) actuator is one of the most commonly applied devices in practical use, such as in micromirrors, microresonators, microswitches, and accelerometers. ${ }^{(1)}$ Owing to the simplicity of the structure, the rapid response, and the low power consumption, parallel-plate-type electrostatic MEM actuators are mostly used. The one-dimensional parallel-plate electrostatic MEM actuator consists of two fixed plates at the top and bottom of the component and a moving plate in an electrical field. Because the capacitance is directly related to the position of the moving plate, a positional sensor that uses capacitance measurements to measure displacements can be developed. ${ }^{(2)}$ The positional regulation of the electrostatic MEM actuator is important in these applications. To solve the control problem of the parallel-plate-type microactuator, both charge control ${ }^{(3-6)}$ and voltage control $^{(7,8)}$ approaches are utilized. In practical applications, voltage control is more easily implemented than charge control. ${ }^{(9)}$

Many studies have been published on voltage control approaches, such as static and dynamic output feedback, ${ }^{(10)}$ nonlinear control, ${ }^{(11)}$ passive control, ${ }^{(12)}$ back-stepping control, ${ }^{(13)}$ gain scheduling $\mathrm{H} \infty$ control, ${ }^{(14)}$ linear parameter-varying control, ${ }^{(15,16)}$ adaptive fuzzy control, ${ }^{(17)}$ an artificial algorithm method, ${ }^{(18)}$ and extended state observer-based control. ${ }^{(9,19)}$ Among these

*Corresponding author: e-mail: 20190207@fjsmu.edu.cn https://doi.org/10.18494/SAM.2020.2918 
studies, in Ref. 10, four output feedback control strategies were investigated by the input-output linearization approach. In Refs. 11 and 13, Lyapunov-based back-stepping design methods were used to propose a nonlinear control scheme for an electrostatic microactuator. In Ref. 14, gain-scheduling $\mathrm{H} \infty$ control by a linearized model was reported, and linear time-varying techniques were applied to the voltage control problem in Refs. 15 and 16. Intelligent control methods, such as adaptive fuzzy control ${ }^{(17)}$ and the artificial algorithm ${ }^{(18)}$ approach, were introduced for the development of control strategies for electrostatic MEM actuators. In Refs. 9 and 19, active disturbance rejection control schemes, which consist of an extended state observer and PD-type control, were developed in the presence of external disturbance and noise.

Compared with previous studies, ${ }^{(10,11,13)}$ the novelty of this study is the introduction of an adaptive two-stage sliding mode control scheme for the positional regulation of a parallel-plate-type electrostatically actuated MEM actuator. There are three steps to develop the control scheme. Firstly, the dynamical model for the MEM actuator is normalized by means of a normalized time-scale approach. Then, a nonlinear state transformation is defined to transform the normalized dynamical model to a nearly controllable canonical form with a nonlinear cubic-power term. An adaptive two-stage sliding mode control scheme based on the nearly controllable canonical form is introduced. In the designed adaptive sliding mode control scheme, two types of sliding function are involved. The level 1 sliding function is defined by the nonlinear transformed states of the dynamical system, where the asymptotic stability of transformed states is inherent. The level 2 sliding function is formed by the level 1 sliding function, with which finite-time stabilization is guaranteed. The proposed sliding mode control scheme also includes time-varying feedback gains, which are updated according to suitable adaptation rules.

This paper is organized as follows. The dynamical model of an electrostatic MEM microactuator is introduced and the normalized time-scale approach and nonlinear state transformation are also discussed in Sect. 2. The design of the control scheme is outlined and the guaranteed stability is proven in Sect. 3. In Sect. 4, numerical studies made to illustrate the effectiveness of the present scheme are reported. Finally, conclusions are given in Sect. 5.

\section{Dynamical Model of Electrostatic Microactuator}

The one-dimensional model of an electrostatic MEM microactuator is shown in Fig. 1. This microactuator has three parallel plates with a middle moving plate (electrode) and top and bottom fixed plates.

In the system, the capacitance of the microactuator is $C(t)=\varepsilon A / y(t)$, where $\varepsilon$ and $A$ are the permittivity and the cross-sectional area of an electrode, respectively. The attractive electrostatic force from the top electrode is $f(t)=Q^{2}(t) / 2 \varepsilon A$, where $Q(t)$ is the charge on an electrode. The dynamical equation of the model depicted in Fig. 1 is given as ${ }^{(20)}$

$$
\begin{aligned}
& \dot{Q}(t)=I(t)=\frac{1}{R}\left(V(t)-\frac{Q(t) y(t)}{\varepsilon A}\right), \\
& M \ddot{y}(t)=-B \dot{y}(t)-K\left(y(t)-y_{0}\right)-\frac{Q^{2}(t)}{2 \varepsilon A},
\end{aligned}
$$




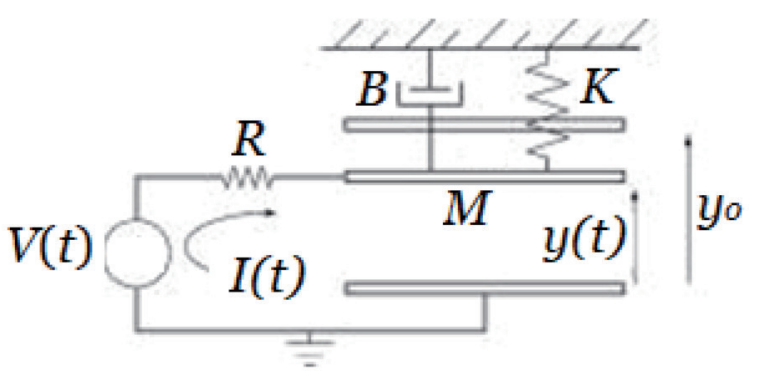

Fig. 1. Model of an electrostatic microactuator.

where $y(t)$ is the gap between the moving and bottom electrodes. $y_{0}$ is the zero-voltage gap. $M, B$, and $K$ are the mass of the moving electrode, the damping ratio, and the linear spring constant, respectively. $I(t)$ and $V(t)$ are the current in the device and the control voltage, respectively. The state vector of the system is $\left[\begin{array}{lll}Q(t) & y(t) & \dot{y}(t)\end{array}\right]^{T} \in R^{3}, y(t) \geq \delta_{0}>0 . \quad \delta_{0}$ denotes the thickness of the insulating layer coated on the bottom electrode; $\delta_{0}$ is helpful in preventing a singularity in the mathematical model. For the accurate measurement of system states, the direct detection of the velocity $\dot{y}(t)$ of the moving electrode is possible. ${ }^{(21)}$ The charge $Q(t)$ on the device can be inferred from the measurements of voltage and capacitance. It is possible for the sensing system to detect the voltage between the moving and bottom electrodes and the capacitance across the device. $^{(22)}$

For convenience in performing the normalized time scaling of Eq. (1), we defined a positive constant $\sigma$ and propose the following normalized terms:

$$
\tau=\sigma t, \quad \alpha=\sigma \varepsilon A R, \quad \beta=\sigma \varepsilon A \sqrt{\sigma M R} .
$$

Moreover, let $u(\tau)=V / \beta R$ be the normalized control input. The normalized state vector for Eq. (1) is chosen as

$$
Z(\tau)=\left[\begin{array}{lll}
z_{1}(\tau) & z_{2}(\tau) & z_{3}(\tau)
\end{array}\right]^{T}=\left[\begin{array}{lll}
Q / \beta & y / \beta & \dot{y} / \beta
\end{array}\right]^{T}
$$

Then, the normalized state equation of an electrostatic MEM microactuator is described as

$$
\begin{aligned}
& \dot{z}_{1}(\tau)=-z_{1}(\tau) z_{2}(\tau)+u(\tau), \\
& \dot{z}_{2}(\tau)=z_{3}(\tau), \\
& \dot{z}_{3}(\tau)=-2 \xi \omega z_{3}(\tau)-\omega^{2}\left(z_{2}(\tau)-\hat{y}_{0}\right)-z_{1}^{2}(\tau) / 2
\end{aligned}
$$

where $\omega_{n}^{2}=K / M, 2 \xi \omega_{n}=B / M, \omega=\omega_{n} / \sigma$, and $\hat{y}_{0}=y_{0} / \alpha$.

The goal of the controlled microactuator is to regulate the state vector $Z(\tau)$ to the constant desired state vector $Z_{d}$. For the system given by Eq. (4), the constant vector $Z_{d}$ is defined as 


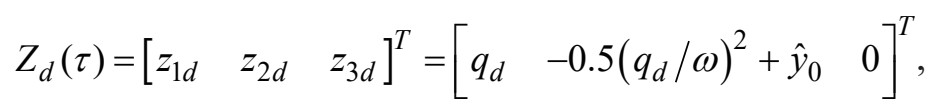

where $z_{1 d}=q_{d}$ is the normalized desired charge. $z_{2 d}$ and $z_{3 d}$ are the normalized desired gap and velocity, respectively. It can be verified that the normalized desired charge is $q_{d}=\sqrt{2 \omega^{2}\left(\hat{y}_{0}-z_{2 d}\right)}$, when the normalized gap $z_{2}(\tau)$ reaches the normalized desired gap $z_{2}=z_{2 d}$. To this end, the control objective of the position-regulating problem for the electrostatic MEM microactuator is to determine an appropriate control scheme $u(\tau)$, such that the error state vector $Z(\tau)-Z_{d}$ converges to zero, that is, $\lim _{\tau \rightarrow \infty}\left(Z(\tau)-Z_{d}\right) \rightarrow 0^{3 \times 1}$, for any initial error state vector $Z(0)-Z_{d}$.

To develop the control scheme of the position-regulating problem, we further consider the following nonlinear state transformation:

$$
X(\tau)=\left[\begin{array}{c}
x_{1}(\tau) \\
x_{2}(\tau) \\
x_{3}(\tau)
\end{array}\right]=\left[\begin{array}{c}
z_{2}(\tau)-z_{2 d} \\
z_{3}(\tau) \\
-2 \xi \omega z_{3}(\tau)-\omega^{2}\left(z_{2}(\tau)-\hat{y}_{0}\right)-0.5 z_{1}^{2}(\tau)
\end{array}\right]
$$

From Eq. (6), it is easy to check that the transformed state vector $X(\tau)$ converging to zero implies that the state vector $Z(\tau)$ converges to the constant desired state vector $Z_{d}$. Thus, the positional regulation of the system given by Eq. (1) is equivalent to the state stabilization problem. That is, $\lim _{\tau \rightarrow \infty} X(\tau) \rightarrow 0^{3 \times 1}$ for any initial transformed state vector $X(0)$.

In addition, by considering the system uncertainty $\Delta(\tau)$ and external disturbance $d(\tau)$, the transformed state equation of the microactuator system can be presented as follows using the state transformation in Eq. (6):

$$
\dot{X}(\tau)=\left[\begin{array}{ccc}
0 & 1 & 0 \\
0 & 0 & 1 \\
0 & -\omega^{2} & -2 \xi \omega
\end{array}\right] X(\tau)+\left[\begin{array}{c}
0 \\
0 \\
x_{1}^{2}(\tau) x_{2}(\tau)+\Delta(t)
\end{array}\right]+\left[\begin{array}{l}
0 \\
0 \\
1
\end{array}\right](U(\tau)+d(\tau)),
$$

where the appended control input is $U(\tau)=-z_{1}(\tau) u(\tau)$.

\section{Design of Control Scheme}

In the following, the rule of thumb for the adaptive two-stage sliding mode control scheme is introduced. There are two basic steps to stabilize the system in Eq. (7). First, two types of sliding function are chosen. The level 1 sliding function $s(\tau)$ is defined by the transformed states $x_{i}(\tau), i=1,2,3$, where the desired stabilization of transformed states is embedded in $s(\tau)=0$ and $\dot{s}(\tau)=0$. The level 2 sliding function $\sigma(\tau)$ is formed by the level 1 sliding function $s(\tau)$, with which the finite-time stabilization of $s(\tau)$ is guaranteed under $\sigma(\tau)=0$ and $\dot{\sigma}(\tau)=0$. 
Secondly, the adaptive sliding mode control law $U(\tau)=U_{e q}(\tau)+U_{s w}(\tau)$ is designed so that $\sigma(\tau)=0$ and $\dot{\sigma}(\tau)=0$; then, any initial value of $s(\tau)$ converges to and remains at the origin even in the presence of system uncertainty $\Delta(\tau)$ and external disturbance $d(\tau)$. When the status of $s(\tau)=0$ is maintained, it means that the trajectory in phase space for the transformed states given by Eq. (6) is stabilized under the embedded converging motion. Then, the system in Eq. (7) is stabilized.

The level 1 sliding function $s(\tau)$ is defined as

$$
s(\tau)=\gamma\left(x_{3}(t)+\int_{0}^{t} \sum_{i=1}^{3} \lambda_{i} x_{i}(\tau) d \tau\right)
$$

where $\gamma$ is the design parameter and $\lambda_{i}, i=1,2,3$ are positive parameters. They are calculated from

$$
\Gamma=\left[\begin{array}{lll}
\lambda_{3} & \lambda_{2} & \lambda_{1}
\end{array}\right]=-B^{T} P
$$

where the symmetric matrix $P$ is obtained by solving the following algebraic Riccati equation:

$$
A^{T} P+P A-P B^{T} B P+Q=O
$$

where

$$
A=\left[\begin{array}{cc}
O_{2 \times 1} & I_{2 \times 2} \\
0 & O_{1 \times 2}
\end{array}\right], \quad B=\left[\begin{array}{lll}
0 & 0 & 1
\end{array}\right]^{T}, \quad Q=\theta I_{3 \times 3} .
$$

$\theta$ is the design parameter and $I_{2 \times 2}$ and $I_{3 \times 3}$ are the identity matrices. When the conditions $s(\tau)=0$ and $\dot{s}(\tau)=0$ are satisfied, $\dot{s}(\tau)=0$ can be expressed as

$$
\dddot{x}_{1}(\tau)+\lambda_{3} \ddot{x}_{1}(\tau)+\lambda_{2} \dot{x}_{1}(\tau)+\lambda_{1} x_{1}(\tau)=0
$$

Because the characteristic equation $c^{3}+\lambda_{3} c^{2}+\lambda_{2} c+\lambda_{1}=0$ is Hurwitz, the asymptotic stability of Eq. (12) is guaranteed.

The level 2 sliding function $\sigma(\tau)$ is defined as

$$
\sigma(\tau)=[s(\tau)]^{p / q}+k \int_{0}^{\tau}[s(\tau)]^{m / q} d \tau
$$

where $k>0, p>q, p>m, m+q>p$, and $p, q, m$ are positive and odd integers. For $\sigma(\tau)=0$ and $\dot{\sigma}(\tau)=0$, the finite-time stability of $s(\tau)$ is obtained as follows. Taking the time derivatives with respect to $\tau$ of Eq. (13) yields 


$$
\frac{p}{q}[s(\tau)]^{(p-q) / q} \cdot \dot{s}(\tau)+k[s(\tau)]^{m / q}=0 .
$$

Letting $\phi(\tau)=[s(\tau)]^{1 / q}$, we obtain

$$
\begin{aligned}
& p[\phi(\tau)]^{p-m-1} \cdot \dot{\phi}(\tau)+k=0 \\
& \Rightarrow \frac{p}{p-m}\left([s(\tau)]^{(p-m) / q}-[s(\tau=0)]^{(p-m) / q}\right)+k \tau=0 .
\end{aligned}
$$

From Eq. (15), there exists a finite time, $\tau=T_{s} \geq 0$, such that the level 1 sliding function $s(\tau)$ moves from $s(0)$ to $s\left(T_{s}\right)=0$. The finite time $T_{s}$ is given by

$$
T_{s}=\frac{p}{k(p-m)}[s(0)]^{(p-m) / q} .
$$

At this point, it is concluded that the object of the control scheme design is to ensure that $\sigma(\tau)=0$ and $\dot{\sigma}(\tau)=0$ are satisfied so that $s(\tau)=0$ approaches the origin in a finite time and remains there. Then, conditions $s(\tau)=0$ and $\dot{s}(\tau)=0$ are satisfied such that the transformed states $x_{i}, i=1,2,3$ tend to zero asymptotically according to Eq. (12).

\section{Theorem}

If the input control $u(\tau)$ in the system in Eq. (7) in the nonlinear feedback control scheme is designed to be

$$
u(\tau)=-U(\tau) / z_{1}(\tau)
$$

the appended control input $U(\tau)$ has the form

$$
\begin{aligned}
U(\tau) & =U_{e q}(\tau)+U_{s w}(\tau) \\
& =-(k q / \gamma p)[s(\tau)]^{(m+p-q) / q}-\left[K_{0}(\tau)+\sum_{i=1}^{3} K_{i}(\tau)\left|x_{i}(\tau)\right|\right] \cdot \sin (\sigma(\tau)),
\end{aligned}
$$

where $s(\tau)$ and $\sigma(\tau)$ are defined in Eqs. (8) and (13), respectively. The positive and adaptive feedback gains $K_{i}(\tau), i=0,1,2,3$ are updated according to the following adaptation algorithms:

$$
\begin{gathered}
\dot{K}_{0}(\tau)=\rho_{0}|\sigma(\tau) \| s(\tau)|^{(p / q)-1} \geq 0, \\
\dot{K}_{i}(\tau)=\rho_{i}\left|x_{i}(\tau)\|\sigma(\tau)\| s(\tau)\right|^{(p / q)-1} \geq 0, i=1,2,3,
\end{gathered}
$$


where $K_{i}(0)=0$ and $\rho_{i}, i=0,1,2,3$ are the positive constants determined in the adaptation process. Then, the level 1 sliding function $\sigma(\tau)$ will be asymptotically stabilized and remain at $\sigma(\tau)=0$ and $\dot{\sigma}(t)=0$. It follows that the level 2 sliding function $s(\tau)$ converges to zero in the finite time $T_{S}$ defined in Eq. (16). When the status of $s(\tau)=0$ is maintained, the trajectory in phase space for the transformed states $x(\tau)_{i}, i=1,2,3$ of the system in Eq. (7) is stabilized in the inherent converging motion given by Eq. (12), where the positive parameters $\lambda_{i}, i=1,2,3$ are obtained from Eqs. (9)-(11). Then, the position regulation control problem of the system in Eq. (4) can be solved.

\section{Proof}

The Lyapunov candidate function is selected to be

$$
V(t)=\frac{1}{2} \sigma^{2}(t)+\sum_{i=0}^{n} \frac{\gamma p}{2 q \rho_{i}}\left(K_{i}(t)-\bar{K}_{i}\right)^{2} \geq 0
$$

where $\bar{K}_{i}, i=0,1,2,3$ are sufficiently large positive constants and satisfy the following inequalities:

$$
\bar{K}_{0}>|\Delta|+|d|>0, \bar{K}_{1}>\lambda_{1}>0, \bar{K}_{2}>\lambda_{2}-\omega^{2}>0, \bar{K}_{1}>\lambda_{3}-2 \xi \omega>0 .
$$

Taking the derivative of Eq. (21) with respect to $\tau$ along with the solutions of the system in Eq. (7), the two sliding functions Eqs. (8) and (13), and the control law in Eq. (17), we obtain

$$
\begin{aligned}
\dot{V}= & \sigma \dot{\sigma}+\sum_{i=0}^{3} \frac{\gamma p}{2 q \rho_{i}}\left(K_{i}-\bar{K}_{i}\right) \dot{K}_{i} \\
=\sigma & \left.k[s]^{(m / q)}+(\gamma p / q)[s]^{(p / q)-1}\left(\lambda_{1} x_{1}+\left(\lambda_{2}-\omega^{2}\right) x_{2}+\left(\lambda_{3}-2 \xi \omega\right) x_{3}+\Delta+d+U\right)\right] \\
& +\sum_{i=0}^{3} \frac{\gamma p}{2 q \rho_{i}}\left(K_{i}-\bar{K}_{i}\right) \dot{K}_{i} .
\end{aligned}
$$

Substituting Eqs. (17)-(20) into Eq. (21) with the criteria in Eq. (22) yields

$$
\begin{aligned}
\dot{V} & =(\gamma p / q)[s]^{(p / q)-1} \sigma\left[\lambda_{1} x_{1}+\left(\lambda_{2}-\omega^{2}\right) x_{2}+\left(\lambda_{3}-2 \xi \omega\right) x_{3}+\Delta+d+\left(K_{0}+\sum_{i=1}^{3} K_{i}\left|x_{i}\right|\right) \cdot \operatorname{sign}(\sigma)\right]+\sum_{i=0}^{3} \frac{\gamma p}{2 q \rho_{i}}\left(K_{i}-\bar{K}_{i}\right) \dot{K}_{i} \\
& \left.\left.\leq(\gamma p / q)|s|^{(p / q)-1}|\sigma| \cdot \cdot\left[\lambda_{1}\left|x_{1}\right|+\left(\lambda_{2}-\omega^{2}\right)\left|x_{2}\right|+\left(\lambda_{3}-2 \xi \omega\right)\left|x_{3}\right|+|\Delta|+|d|+\left(K_{0}+\sum_{i=1}^{3} K_{i} \mid x_{i}\right)\right]\right)\right]+\sum_{i=0}^{3} \frac{\gamma p}{2 q \rho_{i}}\left(K_{i}-\bar{K}_{i}\right) \dot{K}_{i} \\
& \Rightarrow \dot{V} \leq(\gamma p / q)|s|^{(p / q)-1}|\sigma|\left[-\left(\bar{K}_{0}-|\Delta|-|d|\right)-\left(\bar{K}_{1}-\lambda_{1}\right)\left|x_{1}\right|-\left(\bar{K}_{2}-\left(\lambda_{2}-\omega^{2}\right)\right)\left|x_{2}\right|-\left(\bar{K}_{3}-\left(\lambda_{3}-2 \xi \omega\right)\right)\left|x_{3}\right|\right]<0 .
\end{aligned}
$$


Therefore, the condition of $\dot{V}<0$ is satisfied. The level 2 sliding function $\sigma(\tau)$ can reach $\sigma(\tau)=0$ and $\dot{\sigma}(\tau)=0$ asymptotically. Then, the level 1 sliding function $s(\tau)$ approaches zero in the finite time $T_{s}$ given by Eq. (16). The conditions $s(\tau)=0$ and $\dot{s}(\tau)=0$ are satisfied such that the transformed states $x_{i}(\tau), i=1,2,3$ defined in Eq. (12) tend to zero asymptotically. It is thus proven that the positional regulation is accomplished.

\section{Numerical Studies and Discussion}

In the following section, the effectiveness of the proposed adaptive two-stage sliding mode control scheme for the positional regulation of the system in Eq. (4) is verified. The fourth-order Runge-Kutta method is used to implement the closed-loop systems with a time step size of $10^{-4}$ in numerical simulations.

The normalized natural frequency of the system is $\omega=1.0$ and the damping ratio is $\xi=0.01$. The normalized zero voltage gap is taken as $y_{0}=1.0$. The positional regulation of the system in Eq. (4) is performed for $z_{2 d}=0.6$. The initial conditions of the system in Eq. (4) are chosen as $z_{1}(0)=0.25, z_{2}(0)=1.0$, and $z_{3}(0)=0$. For the adaptive two-stage sliding mode control scheme in Eq. (18) associated with Eqs. (8)-(11), (13), (19), and (20), the positive constants are set as $\gamma=0.75$, $\theta=0.2, p=11, q=7, m=9, k=2, \rho_{0}=10, \rho_{1}=40$, and $\rho_{2}=\rho_{3}=125$. It is presumed that the system uncertainty and external disturbance are $\Delta(\tau)=0.1 \sin \left(\sqrt{z_{1}^{2}(\tau)+z_{2}^{2}(\tau)+z_{3}^{2}(\tau)}\right)$ and $d(\tau)$ $=0.05 \cos (\pi \tau)$, respectively. In the case of the normalized desired gap, $z_{2 d}=0.6$, the normalized desired charge is $z_{1 d}=\sqrt{0.8}=0.8944$. The time responses for the level 1 and 2 sliding mode functions and the state trajectory of the controlled system in the phase plane $(s(\tau), \sigma(\tau))$ are shown in Fig. 2. It is shown that $\sigma(\tau)$ converges to zero within a finite time; thus, $s(\tau)=0$ and $\dot{s}(\tau)=0$ are realized after $\sigma(\tau)=0$ and $\dot{\sigma}(\tau)=0$.
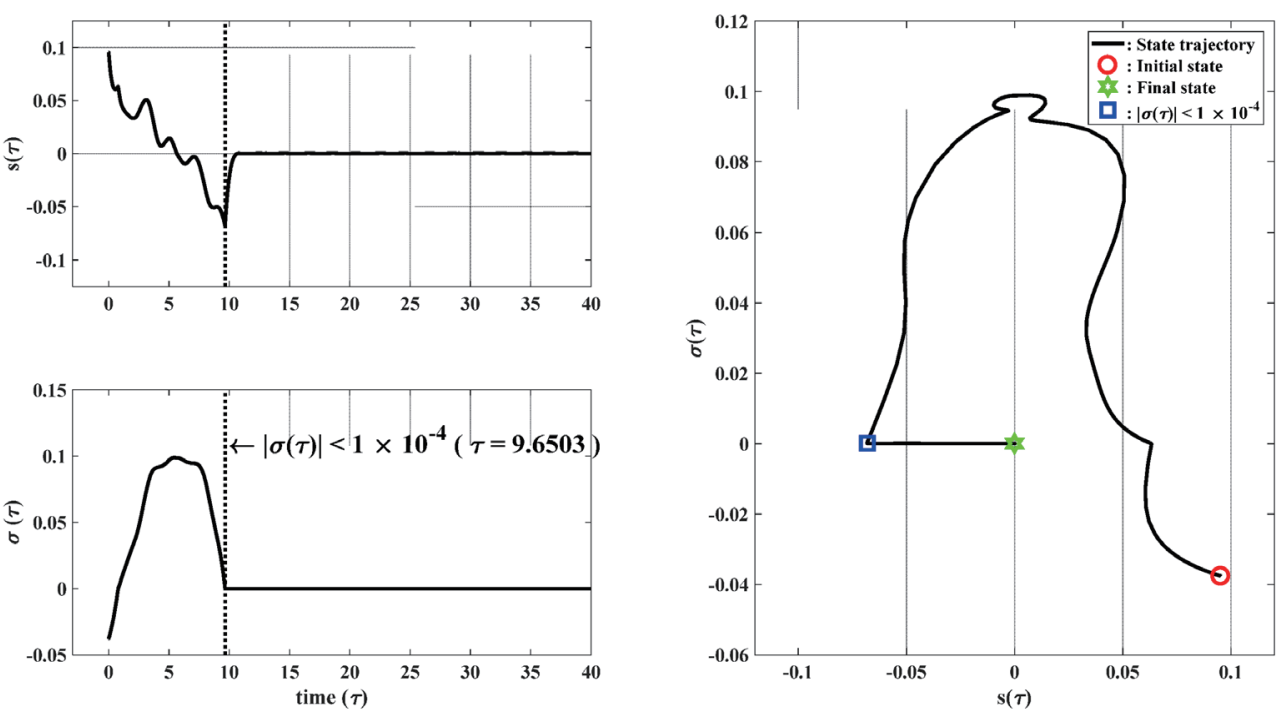

Fig. 2. (Color online) Time responses of $s(\tau), \sigma(\tau)$, and state trajectory in $(s(\tau), \sigma(\tau))$ plane for $z_{2 d}=0.6$. 
Figure 3 shows the time responses of the adaptive feedback gains $K_{i}(\tau), i=0,1,2,3$ and the control input $u(\tau)$. When compared with the past work ${ }^{(23)}$ for the robust controller design of an electrostatic MEM actuator, the proposed control input $u(\tau)$ is clearly continuous and chatter-free in the appearance of system uncertainties and external disturbances. Figure 4 demonstrates the time responses of transformed states $x_{i}(\tau), i=1,2$, 3. It is clear that the system in Eq. (7) is stabilized. Figure 5 depicts the time responses of the normalized charge, $z_{1}(\tau)$, the normalized gap $z_{2}(\tau)$, and the normalized velocity $z_{3}(\tau)$. When $z_{2}(\infty) \rightarrow z_{2 d}=0.6$ is achieved, the problem of the positional regulation of the system in Eq. (4) is solved.

In the other case of the normalized desired gap, $z_{2 d}=0.2$, the normalized desired charge is $z_{1 d}=\sqrt{1.6}=1.2649$. Figure 6 depicts the time responses of $s(\tau), \sigma(\tau)$, and the state trajectory of the controlled system in the $s(\tau)$ vs $\sigma(\tau)$ plane. In Fig. 7, it is shown that the positional regulation of the system in Eq. (4) is completed for $z_{2}(\infty) \rightarrow z_{2 d}=0.2$.
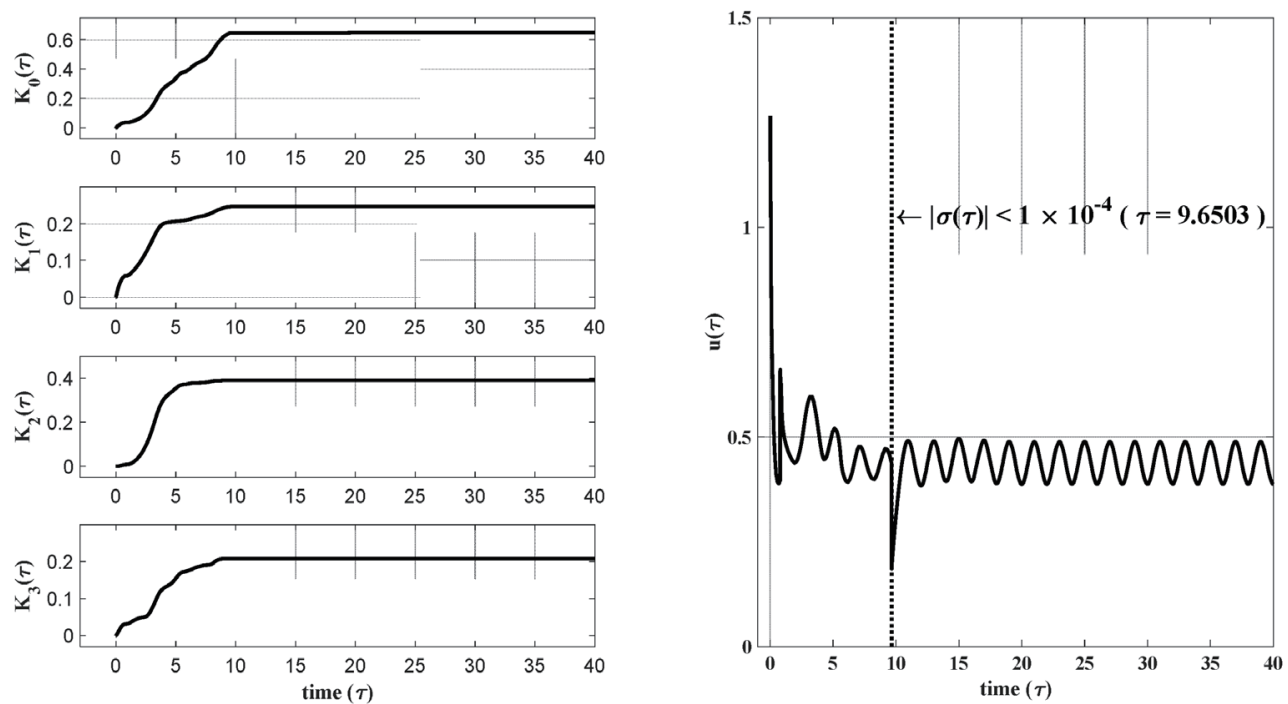

Fig. 3. Time responses of $K_{i}(\tau), i=0,1,2,3$ and $u(\tau)$ for $z_{2 d}=0.6$.
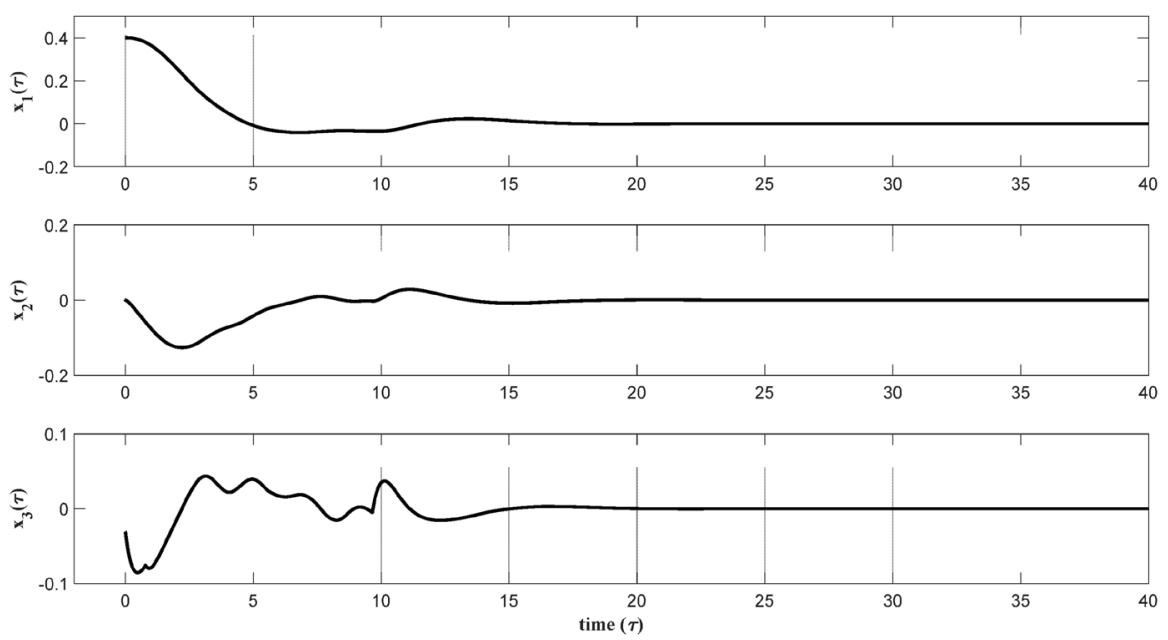

Fig. 4. Time responses of $x_{i}(\tau), i=1,2,3$ for $z_{2 d}=0.6$. 


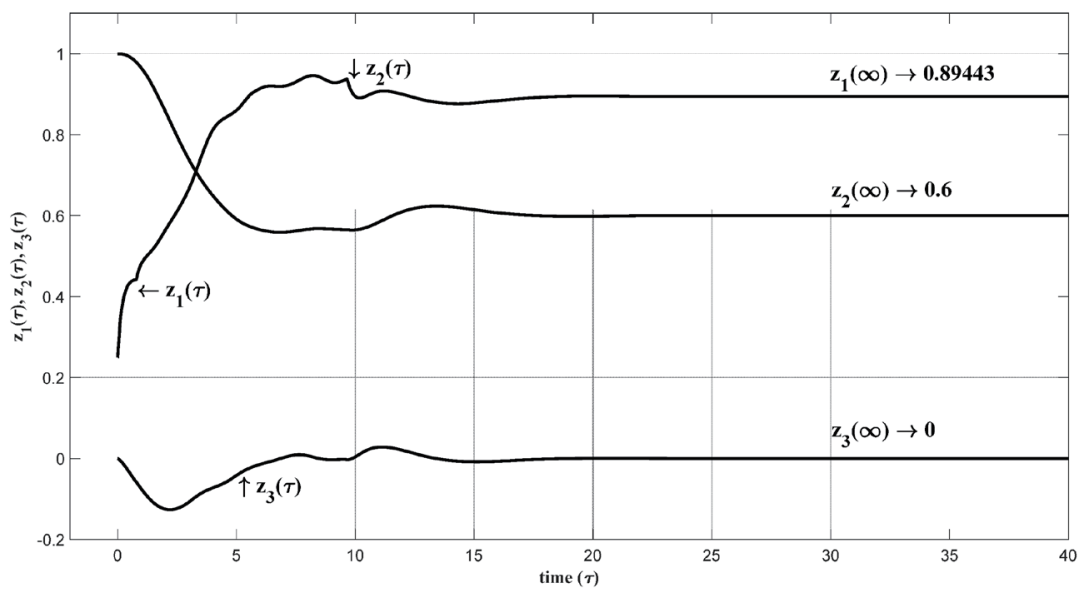

Fig. 5. Time responses of $z_{i}(\tau), i=1,2,3$ for $z_{2 d}=0.6$.
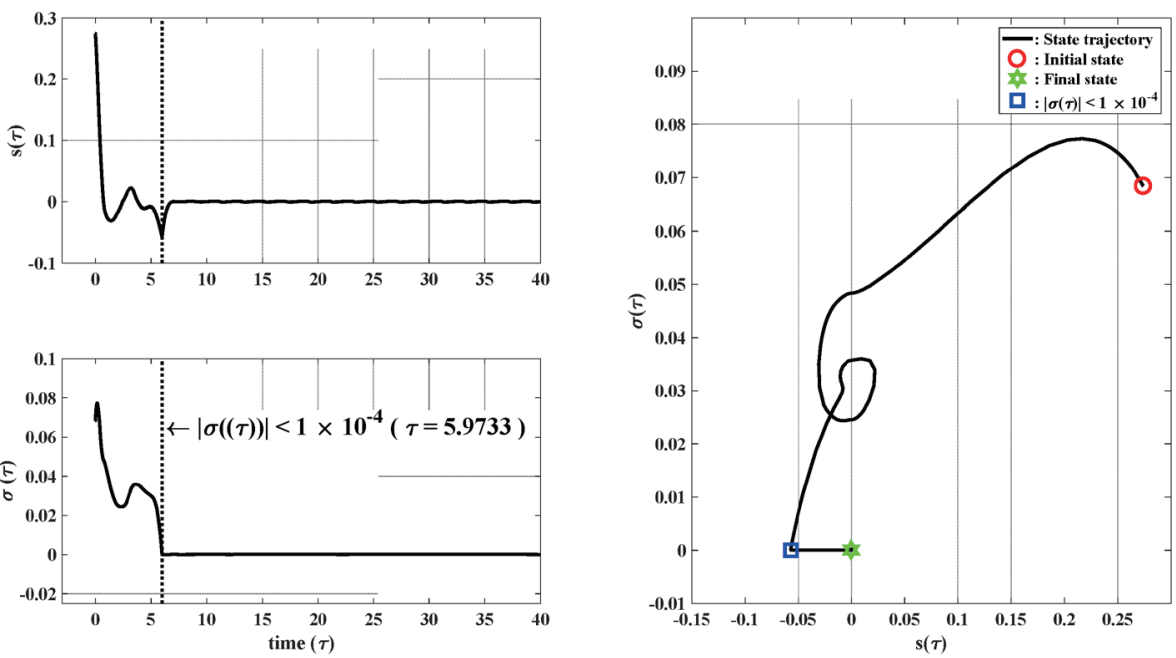

Fig. 6. (Color online) Time responses of $s(\tau), \sigma(\tau)$, and state trajectory in $(s(\tau), \sigma(\tau))$ plane for $z_{2 d}=0.2$.

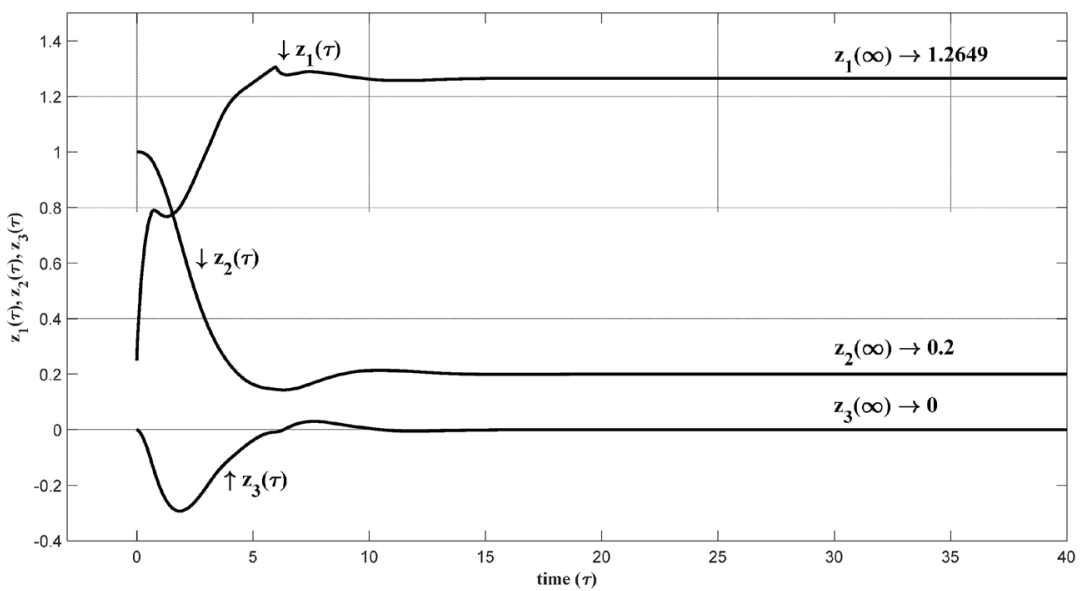

Fig. 7. Time responses of $z_{i}(\tau), i=1,2,3$ for $z_{2 d}=0.2$. 
The proposed control scheme can be implemented similarly to those in previous studies. ${ }^{(21,22)}$ The direct detection of the velocity $\dot{y}(t)$ for the moving electrode is possible by the accurate measurement of system states. ${ }^{(21)}$ The charge $Q(t)$ on the MEM actuator can be inferred from the measurements of voltage and capacitance. The sensing system can be set to sense the voltage between the moving and bottom electrodes, and the capacitance across the device. ${ }^{(22)}$

\section{Conclusion}

In this study, an adaptive two-stage sliding mode control scheme for achieving the positional regulation of an electrostatic MEM actuator has been proposed. To develop the control scheme, a normalized time-scale approach and a nonlinear state transformation are applied to the dynamical model. It is proved that the proposed control scheme can guarantee the regulation of the states of the system in Eq. (4) to the desired values. Numerical studies are performed for two cases to demonstrate the effectiveness of the presented control scheme.

\section{Acknowledgments}

This work was carried out as part of the Intelligent Manufacturing Program coordinated by the Beautiful China Research Institute of Sanming University with financial support from the Operational Funding of the Advanced Talents for Scientific Research (19YG04 and 19YG05) of Sanming University and the Science and Technology Department of Fujian Province (Grant no. 2017H6019). The authors also acknowledge support from the College of Mechanical and Electrical Engineering of Sanming University.

\section{References}

1 W. C. Chuang, H. L. Lee, P. Z. Chang, and Y. C. Hu: Sensors 10 (2010) 6149. https://doi.org/10.3390/ s100606149

2 M. S.-C. Lu and G. K. Fedder: J. Microelectromech. Syst. 13 (2004) 759. https://doi.org/10.1109/ JMEMS.2004.835761

3 R. A. Guardia, A. Dehe, R. Aigner, and L. M. Castaner: J. Microelectromech. Syst. 11 (2002) 255. https://doi. org/10.1109/JMEMS.2002.1007404

4 J. I. Seeger and B. E. Boser: J. Microelectromech. Syst. 12 (2003) 656. https://doi.org/10.1109/ JMEMS.2003.818455

5 G. Zhu, J. Penet, and L. Saydy: ASME J. Dyn. Syst. Meas. Control 129 (2007) 786. https://doi. org/10.1115/1.2789469

6 M. H. Salah, A. M. Al-Jarrah, and E. Tatlicioglu: Proc. 2011 Int. Conf. Control and Applications (CA, 2011). https://doi.org/10.2316/P.2011.729-105

7 L. A. Rocha, E. Cretu, and R. F. Wolffenbuttel: J. Microelectromech. Syst. 15 (2006) 69. https://doi.org/10.1109/ JMEMS.2005.859099

8 G. Zhu, J. Levine, L. Praly, and Y. A. Peter: J. Microelectromech. Syst. 15 (2006) 1165. https://doi.org/10.1109/ jmems.2006.880198

9 P. Kandula, and L. Dong: ASME J. Dyn. Syst. Meas. Control 140 (2018) 061012-1. https://doi. org/10.1115/1.4038943

10 D. H. S. Maithripala, J. M. Berg, and W. P. Dayawansa: ASME J. Dyn. Syst. Meas. Control 127 (2005) 443. https://doi.org/10.1115/1.1985443

11 G. Zhu, J. Levine, and L. Praly: Proc. 2005 IEEE 44th Conf. Decision and Control and European Control Conf. (CDC-ECC, 2005) 7534. https://doi.org/10.1109/CDC.2005.1583377 
12 I. P. M. Wickramasinghe, D. H. S. Maithripala, B. D. Kawade, J. M. Berg, and W. P. Dayawansa: IEEE Trans. Control Syst. Technol. 17 (2009) 249. https://doi.org/10.1109/TCST.2008.924571

13 M. H. Salah, K. M. Alwidyan, E. Tatlicioglu, and D. M. Dawson: Proc. 2010 IEEE 49th Conf. Decision and Control (CDC, 2010).

14 M. Vagia and A. Tzes: Proc. 2009 American Control Conf. (ACC, 2009) 4805.

15 F. A. Shirazi, J. M. Velni, and K. M. Grigoriadis: J. Microelectromech. Syst. 20 (2011) 302. https://doi. org/10.1109/JMEMS.2010.2090651

16 H. Alwi, A. Zolotas, C. Edwards, and K. Grigoriadis: Proc. 2012 American Control Conf. (ACC, 2012$) 875$. https://doi.org/10.1109/ACC.2012.6314803

17 G. Rigatos, G. Zhu, H. Yousef, and A. Boulkroune: Fuzzy Sets Syst. 290 (2016) 138. https://doi.org/10.1016/ j.fss.2015.08.027

18 A. K. Cevher, A. Yildiz, and A. Akdagli: Microsyst. Tech. 24 (2018) 2137. https://doi.org/10.1007/s00542-0173613-4

19 L. Dong and J. Edward: Proc. 2010 American Control Conf. (ACC, 2012) 3409. https://doi.org/10.1109/ ACC.2010.5531118.

20 S. D. Senturia: Microsystem Design (Springer Science and Business Media, New York, 2001). https://doi. org $/ 10.1007 / \mathrm{b} 117574$

21 H. Toshiyoshi, M. Mita, and H. Fujita: J. Microelectromech. Syst. 11 (2002) 648. https://doi.org/10.1109/ JMEMS.2002.805054

22 F. Ayela, J. L. Bret, J. Chaussy, T. Fournier, and E. Menegaz: Rev. Sci. Instrum. 71 (2000) 2211. https://doi. org/10.1063/1.1150608

23 L. Dong and J. Edwards: Inter. J. Control Sci. Eng. 3 (2013) 8. https://doi.org/10.5923/j.control.20130301.02 